\title{
Cerebral involvement in COVID-19 is associated with metabolic and coagulation derangements: an EEG study
}

\author{
Giordano Cecchetti ${ }^{1,2,3}$ (D) Marco Vabanesi ${ }^{1,2}\left(\right.$ Raffaella Chieffo $^{1}\left({ }^{10} \cdot\right.$ Giovanna Fanelli $^{1} \cdot$ Fabio Minicucci $^{1}$. \\ Federica Agosta ${ }^{3,4} \cdot$ Moreno Tresoldi $^{5} \cdot$ Alberto Zangrillo $^{4,6}$ (1) Massimo Filippi ${ }^{1,2,3,4}$ (D)
}

Received: 6 May 2020 / Revised: 26 May 2020 / Accepted: 28 May 2020 / Published online: 15 June 2020

(c) Springer-Verlag GmbH Germany, part of Springer Nature 2020

Dear Sirs,

In previous reports, clinical characteristics of SARS-CoV-2 infection have been described, and risk factors for the development of acute respiratory distress syndrome (ARDS) and death have been proposed: among them, older age, comorbidities such as hypertension and diabetes mellitus, neutrophilia and markers of end-organ and coagulation impairment [1].

Although mortality due to COVID-19 is mainly linked to visceral multi-organ failure, cerebral involvement might worsen the clinical picture. Up to $36 \%$ of patients with COVID-19 appear to have neurological manifestations, ranging from headache and dizziness to impaired consciousness and cerebrovascular events [2]. The EEG correlates of such manifestations are still not clear.

Giordano Cecchetti and Marco Vabanesi contributed equally to the article and should be both considered first authors.

Electronic supplementary material The online version of this article (https://doi.org/10.1007/s00415-020-09958-2) contains supplementary material, which is available to authorized users.

Massimo Filippi

filippi.massimo@hsr.it

1 Neurophysiology Unit, IRCCS San Raffaele Scientific Institute, Milan, Italy

2 Neurology Unit, IRCCS San Raffaele Scientific Institute, Milan, Italy

3 Neuroimaging Research Unit, Institute of Experimental Neurology, Division of Neuroscience, IRCCS San Raffaele Scientific Institute, Milan, Italy

4 Vita-Salute San Raffaele University, Via Olgettina, 60, 20132 Milan, Italy

5 General Medicine and Advanced Care Unit, IRCCS San Raffaele Scientific Institute, Milan, Italy

6 Anesthesia and Intensive Care Unit, IRCCS San Raffaele Scientific Institute, Milan, Italy
We report a series of 18 consecutive COVID-19 patients, admitted to our Institution between March and April 2020, evaluated with basal electroencephalogram (EEG) to investigate infection-related neurologic manifestations. All patients had their COVID-19 diagnosis confirmed by means of duplo SARS-CoV-2 real-time polymerase chain reaction (RT-PCR) on nasopharyngeal swabs, and compatible chest X-ray and/ or CT scan.

Patients were divided in three groups, according to the level of EEG background activity alteration on a threegrade scale [normal/mild $(N=5)$, moderate $(N=9)$ or severe $(N=4)$ ] derived with modifications from Amodio et al. [3]. Neurological manifestations prompting EEG examination were transient loss of consciousness, seizures/spasms, delirium and coma, with different distribution in the patient groups; no subjects reported history of anosmia/ageusia.

Demographic and clinical features of patients are reported in Table 1. The frequency of focal central nervous system (CNS) lesions at neuroimaging (CT and/or MRI scan) did not differ among groups. We observed generalized EEG slowing in 16/18 (88.9\%) patients; an anterior (bifrontal) prevalence of slow waves was noted in $10 / 18(55.6 \%)$ cases (Fig. S1), a finding observed also in metabolic encephalopathies [4]. Its presence and severity correlated with the degree of EEG alteration ( $p=0.02$, linear regression model). This kind of abnormalities has been recently observed also by Helms [5]. Only two patients presented with epileptiform discharges (one with operated high-grade glioma, one with coma and severe hypernatremia); no seizures were detected.

According to clinical protocols, only one subject in our cohort underwent lumbar puncture to rule out acute encephalitis, with normal results (cells, protein and glucose) and negative RT-PCR test for SARS-CoV-2; in addition, bacterial and virologic assays were negative. In the remaining 17 subjects, lumbar puncture was not performed, since not indicated on the basis of clinical judgement. 
Table 1 Demographical data and main clinical results in patients stratified according to EEG alteration severity

\begin{tabular}{|c|c|c|c|c|c|}
\hline & $\begin{array}{l}\text { Normal/mild EEG } \\
\text { alteration }(N=5)\end{array}$ & Moderate EEG alteration $(N=9)$ & $\begin{array}{l}\text { Severe EEG } \\
\text { alteration } \\
(N=4)\end{array}$ & $p$ value & Estimate $\pm \mathrm{SE}$ \\
\hline Sex: $M / F$ & $4 / 1$ & $5 / 4$ & $2 / 2$ & $0.60^{\mathrm{a}}$ & - \\
\hline Age (years) & $62.4 \pm 15.9$ & $70.4 \pm 8.8$ & $64.3 \pm 4.5$ & $0.34^{\mathrm{a}}$ & - \\
\hline Comorbidities & & & & & - \\
\hline Hypertension & $3 / 5(60.0 \%)$ & $6 / 9(66.7 \%)$ & $2 / 4(50.0 \%)$ & $0.86^{\mathrm{a}}$ & \\
\hline Diabetes & $2 / 5(40.0 \%)$ & $2 / 9(22.2 \%)$ & - & $0.38^{\mathrm{a}}$ & \\
\hline Obesity & - & $1 / 9(11.1 \%)$ & $1 / 4(25.0 \%)$ & $0.51^{\mathrm{a}}$ & \\
\hline CKD & $1 / 5(20.0 \%)$ & $1 / 9(11.1 \%)$ & - & $0.65^{\mathrm{a}}$ & \\
\hline COPD & - & $1 / 9(11.1 \%)$ & $1 / 4(25.0 \%)$ & $0.51^{\mathrm{a}}$ & \\
\hline Asthma & - & $1 / 9(11.1 \%)$ & $1 / 4(25.0 \%)$ & $0.51^{\mathrm{a}}$ & \\
\hline Atrial fibrillation & $1 / 5(20.0 \%)$ & $2 / 9(22.2 \%)$ & $2 / 4(50.0 \%)$ & $0.55^{\mathrm{a}}$ & \\
\hline Other & - & $\begin{array}{l}\text { IHD (2) } \\
\text { Lung cancer (1) }\end{array}$ & - & - & \\
\hline Focal CNS lesions at neuroimaging & $\begin{array}{l}2 / 5(40.0 \%) \\
\text { PRES (1) } \\
\text { Remote IPH (1) }\end{array}$ & $\begin{array}{l}4 / 9(44.4 \%) \\
\text { Glioblastoma (1) } \\
\text { Brain metastasis (1) } \\
\text { Traumatic SDH (1) } \\
\text { Remote hemispheric ischemic lesion } \\
\text { (1) }\end{array}$ & $\begin{array}{l}\text { 1/4 }(25.0 \%) \\
\text { Anterior } \\
\text { pontine } \\
\text { demyelinat- } \\
\text { ing les. (1) }\end{array}$ & $0.81^{\mathrm{a}}$ & \\
\hline Anosmia/ageusia & - & - & - & - & \\
\hline Disease onset-to-door (d) & $2.5 \pm 3.0$ & $5.8 \pm 4.8$ & $6.7 \pm 2.5$ & $0.30^{\mathrm{a}}$ & - \\
\hline Disease onset-to-EEG (d) & $6.6 \pm 7.6$ & $13.3 \pm 11.7$ & $11.0 \pm 9.9$ & $0.56^{\mathrm{a}}$ & - \\
\hline $\mathrm{SpO}_{2}$ at arrival (\%) & $91 \pm 4$ & $86 \pm 13$ & $66 \pm 8$ & $0.05^{\mathrm{b} *}$ & $-0.040(0.019)$ \\
\hline Invasive ventilation & - & $3 / 9(33.3 \%)$ & $3 / 4(75.0 \%)$ & $0.07^{\mathrm{a}}$ & - \\
\hline Body temperature $\left({ }^{\circ} \mathrm{C}\right)$ & $37.3 \pm 1.5$ & $36.8 \pm 0.7$ & $37.9 \pm 0.5$ & $0.49^{\mathrm{a}}$ & \\
\hline Heart rate (bpm) & $91 \pm 12$ & $91 \pm 16$ & $93 \pm 24$ & $0.75^{\mathrm{a}}$ & \\
\hline \multicolumn{6}{|l|}{ Outcome } \\
\hline Alive, discharged & $2 / 5(40.0 \%)$ & $1 / 9(11.1 \%)$ & - & $0.17^{\mathrm{a}}$ & - \\
\hline Alive, in-hospital & $2 / 5(40.0 \%)$ & $4 / 9(44.4 \%)$ & $1 / 4(25.0 \%)$ & & \\
\hline Deceased & $1 / 5(20.0 \%)$ & $4 / 9(44.4 \%)$ & $3 / 4(75.0 \%)$ & & \\
\hline Indication for EEG & $\begin{array}{l}\text { Transient LOC: } 2 / 5 \\
\text { Seizure/spasms: } 2 / 5 \\
\text { Delirium: } 1 / 5 \\
\text { - }\end{array}$ & $\begin{array}{l}\text { Transient LOC: } 3 / 9 \\
\text { Seizure/spasms: } 3 / 9 \\
\text { Delirium: } 1 / 9 \\
\text { Coma: } 2 / 9\end{array}$ & $\begin{array}{l}- \\
- \\
\text { Delirium: 1/4 } \\
\text { Coma: 3/4 }\end{array}$ & $0.03^{\mathrm{a}} *$ & - \\
\hline \multicolumn{6}{|l|}{ EEG features } \\
\hline \multicolumn{6}{|l|}{$\begin{array}{l}\text { Anterior prevalence of diffuse slow } \\
\text { waves }\end{array}$} \\
\hline Absent & $5 / 5(100.0 \%)$ & $3 / 9(33.3 \%)$ & $1 / 4(25.0 \%)$ & $0.02^{\mathrm{b} *}$ & $0.56 \pm 0.22$ \\
\hline Intermittent & - & $3 / 9(33.3 \%)$ & - & & \\
\hline Continuous & - & $3 / 9(33.3 \%)$ & $3 / 4(75.0 \%)$ & & \\
\hline Focal slowing & $1 / 5(20.0 \%)$ & $5 / 9(55.6 \%)$ & $1 / 4(25.0 \%)$ & $0.37^{\mathrm{a}}$ & \\
\hline Epileptiform discharges & - & $1 / 9(11.1 \%)$ & $1 / 4(25.0 \%)$ & $0.51^{\mathrm{a}}$ & \\
\hline Seizures & - & - & - & - & \\
\hline \multicolumn{6}{|l|}{ Laboratory parameters } \\
\hline WBC count $\left(10^{9} / \mathrm{L}\right)$ & $9.52 \pm 5.64$ & $10.79 \pm 6.59$ & $17.58 \pm 2.07$ & $0.045^{\mathrm{b}} *$ & $0.071 \pm 0.033$ \\
\hline Ly count $\left(10^{9} / \mathrm{L}\right)$ & $1.24 \pm 0.38$ & $0.92 \pm 0.52$ & $0.93 \pm 0.57$ & $0.43^{\mathrm{b}}$ & - \\
\hline Hyper/hyponatremia & $1 / 5(20.0 \%)$ & $6 / 9(66.7 \%)$ & $3 / 4(75.0 \%)$ & $0.05^{\mathrm{b} *}$ & $0.82 \pm 0.40$ \\
\hline Creatinine (mg/dL) & $1.43 \pm 0.20$ & $1.52 \pm 1.21$ & $3.35 \pm 2.07$ & $0.024^{\mathrm{b}} *$ & $1.06 \pm 0.42$ \\
\hline $\mathrm{CRP}(\mathrm{mg} / \mathrm{L})$ & $46.5 \pm 19.5$ & $130.8 \pm 81.7$ & $114.6 \pm 101.9$ & $0.14^{\mathrm{b}}$ & - \\
\hline LDH (U/L) & $352 \pm 86$ & $397 \pm 154$ & $436 \pm 174$ & $0.57^{\mathrm{b}}$ & - \\
\hline
\end{tabular}


Table 1 (continued)

\begin{tabular}{|c|c|c|c|c|c|}
\hline & $\begin{array}{l}\text { Normal/mild EEG } \\
\text { alteration }(N=5)\end{array}$ & Moderate EEG alteration $(N=9)$ & $\begin{array}{l}\text { Severe EEG } \\
\text { alteration } \\
(N=4)\end{array}$ & $p$ value & Estimate \pm SE \\
\hline CK (U/L) & $130 \pm 74$ & $428 \pm 927$ & $1827 \pm 3319$ & $0.39^{\mathrm{b}}$ & - \\
\hline Albumin $(\mathrm{g} / \mathrm{L})$ & $25.4 \pm 1.9$ & $26.5 \pm 5.5$ & $24.7 \pm 4.0$ & $0.82^{\mathrm{b}}$ & - \\
\hline PT ratio & $1.08 \pm 0.09$ & $1.16 \pm 0.25$ & $1.68 \pm 0.57$ & $0.031^{\mathrm{b}} *$ & $1.12 \pm 0.47$ \\
\hline APTT ratio & $1.01 \pm 0.05$ & $0.99 \pm 0.08$ & $1.51 \pm 0.43$ & $0.037^{\mathrm{b}} *$ & $1.58 \pm 0.69$ \\
\hline D-dimer $(\mu \mathrm{g} / \mathrm{dL})$ & $1.86 \pm 0.49$ & $2.15 \pm 1.98$ & $12.19 \pm 9.58$ & $0.028^{\mathrm{b}} *$ & $0.064 \pm 0.025$ \\
\hline
\end{tabular}

Values are mean $\pm \mathrm{SD}$ or $N(\%)$

$C K D$ chronic kidney disease, $C O P D$ chronic obstructive pulmonary disease, $I H D$ ischemic heart disease, $S D H$ subdural hematoma, $d$ days, $L O C$ loss of consciousness, WBC white blood cells, $L y$ lymphocyte, $C R P$ C-reactive protein, $L D H$ lactate dehydrogenase, $C K$ creatine kinase, $P R E S$ posterior reversible encephalopathy syndrome, $P T$ prothrombin time, APTT activated partial prothrombin time

${ }^{a}$ Kruskal-Wallis rank sum test

${ }^{b}$ Univariate linear regression model of the specified parameter on the level of EEG alteration (normal, mild, moderate, severe); the estimate of effect $( \pm$ SE), when significant, is shown on the right column. For CK, log transformation was used.

We visually screened vital and laboratory parameters for association with the degree of EEG alteration. Univariate linear regression models were built to evaluate the relationship between patient groups and selected parameters. Among vital parameters (including heart rate and body temperature), only oxygen saturation on room air $\left(\mathrm{SpO}_{2}\right)$, measured at hospital admission appeared to be associated to the EEG abnormalities (Table 1, Fig. 1). As to laboratory parameters, a linear relationship with the severity of EEG alteration was observed for white blood cell (WBC) count, the presence of plasma sodium alterations, serum creatinine, and coagulation parameters (prothrombin time, activated partial thromboplastin time, and D-dimer) (Table 1, Fig. 1).

We recorded for all but three patients the antibiotic and/ or antiviral drugs administered at the time of EEG. We did not observe significant effects of drug-related toxicity in our cohort. Moreover, laboratory parameters most commonly associated to drug therapies did not appear to influence EEG alteration; association with creatinine may be correlated to aggressive diuretic therapy salvage protocols.
Our findings suggest variable degrees of CNS involvement in COVID-19 patients. The presence of focal brain lesions did not appear to impact significantly on EEG background activity. Subjects with laboratory findings which have been associated to poor prognosis [1] showed more frequent CNS impairment, as reflected by EEG alterations. Moreover, lower values of oxygen saturation at admission were associated with more severe EEG abnormalities, suggesting that higher levels of hypoxemia, and possibly longer periods before access to cures, may contribute to brain dysfunction.

Electroencephalogram (EEG) may therefore represent a useful tool to evaluate early cerebral involvement in COVID19 , especially in severe cases; a frequent finding in our cohort was an anterior prevalence of slow waves. We hypothesize that the main drivers of EEG alterations in these patients are metabolic and/or hypoxic encephalopathy, even if our data cannot exclude the contribution of cerebral microangiopathy, yet to be demonstrated. We believe that our data raise a warning against a delayed access to specialized cures in SARS-CoV-2 infection, in order to reduce the incidence of brain suffering. 

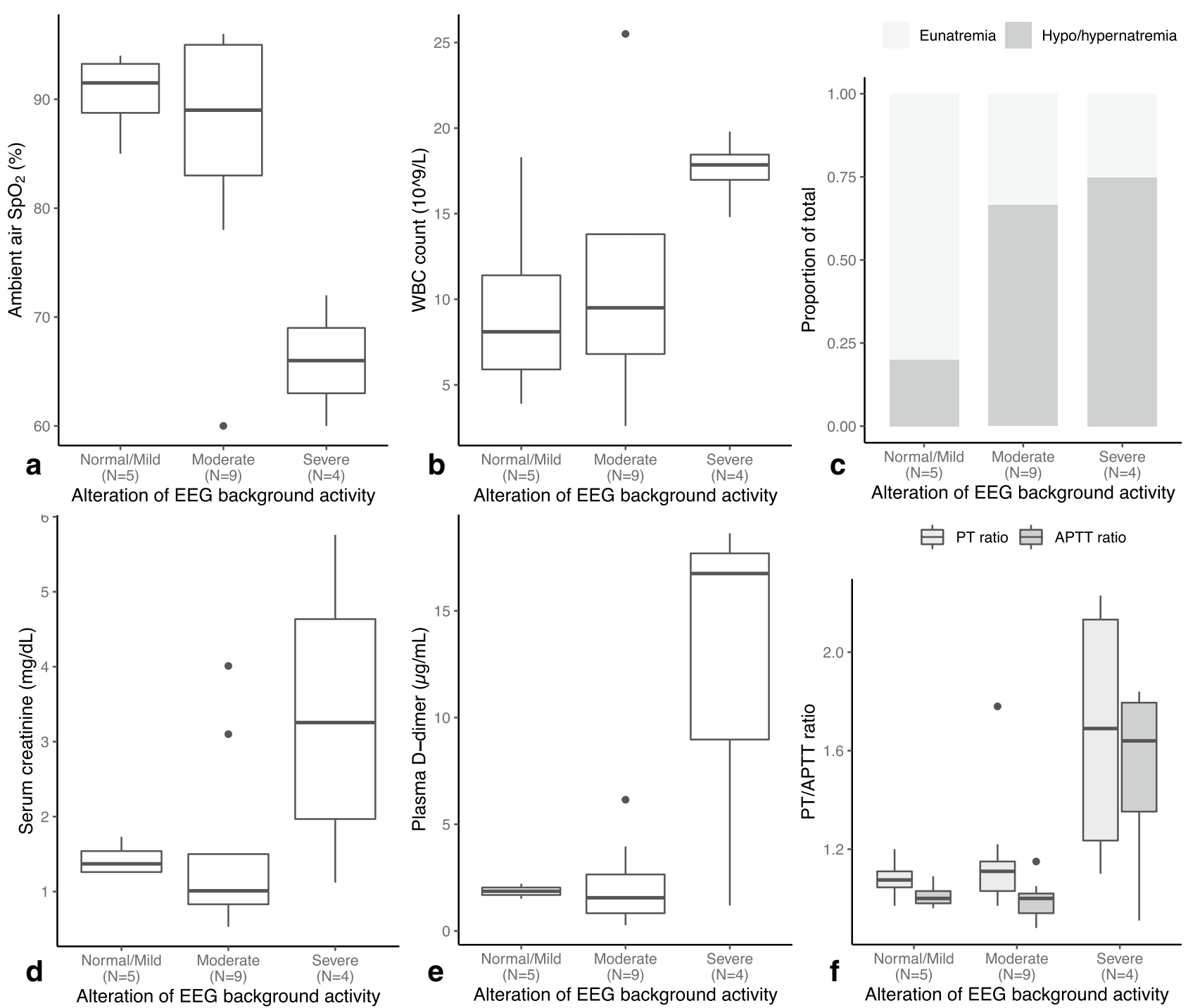

Fig. 1 Plots of vital and laboratory parameters stratified by level of EEG background activity alteration. Only parameters with significant relationship at linear regression model (Table 1) are shown. $\mathrm{SpO}_{2}$

Author contributions GC and MV equally contributed to study conception, data collection, analysis and interpretation and paper drafting. GF contributed to data collection, analysis and interpretation. RC, FA, MT, and AZ contributed to data analysis and interpretation. FM supervised data collection, analysis and interpretation. MF contributed to study conception, data analysis and interpretation. All authors critically reviewed the paper.

Funding Not applicable.

\section{Compliance with ethical standards}

Conflicts of interest No relevant competing interest was declared by the authors. oxygen saturation on room air, $W B C$ white blood cells, $P T$ prothrombin time, $A P T T$ activated partial prothrombin time

Ethics approval Ethical approval was obtained from San Raffaele Hospital Ethics Committee.

Consent to participate Informed consent was obtained from all capable individual participants included in the study as per ethics committee approval.

Availability of data and material Not applicable.

Code availability Not applicable. 


\section{References}

1. Wu C, Chen X, Cai Y, Xia J, Zhou X, Xu S et al (2020) Risk factors associated with acute respiratory distress syndrome and death in patients with coronavirus disease 2019 pneumonia in Wuhan, China. JAMA Intern Med (Epub 2020 Mar 13)

2. Mao L, Jin H, Wang M, Hu Y, Chen S, He Q et al (2020) Neurologic manifestations of hospitalized patients with coronavirus disease 2019 in Wuhan, China. JAMA Neurol (Epub 2020 Apr 10)
3. Amodio P, Marchetti P, Del Piccolo F, de Tourtchaninoff M, Varghese P, Zuliani G et al (1999) Spectral versus visual EEG analysis in mild hepatic encephalopathy. Clin Neurophysiol 110(8):1334-1344

4. Parsons-Smith BG, Summerskill WH, Dawson AM, Sherlock $S$ (1957) The electroencephalograph in liver disease. Lancet 273(7001):867-871

5. Helms J, Kremer S, Merdji H, Clere-Jehl R, Schenck M, Kummerlen C, et al (2020) Neurologic features in severe SARS-CoV-2 infection. N Engl J Med (Epub 2020 Apr 15) 